Helgoländer wiss. Meeresunters. 17, 25-43 (1968)

\title{
Hauptquellen häuslicher Abwässer und deren Bedeutung für die Verunreinigung der Nordsee ${ }^{1}$
}

\author{
Hermann Kayser \\ Biologische Anstalt Helgoland, Mceresstation, Helgoland
}

\begin{abstract}
Major sources of domestic sewages and their significance in the pollution of the North Sea. Domestic wastes are characterized by a high percentage of $\mathrm{C}-, \mathrm{N}$ - and P-containing substances and organic compounds such as vitamins and amino-acids. These substances are of great importance in regard to the metabolism of the sea and may have negative as well as positive effects on the biocoenosis. The sources of domestic wastes can be divided into: (a) effluents from cities or villages located directly at the sea, and (b) river water which carries more or less decomposed domestic sewages released at various places in the interior part of the country. The degree of purification of waste water, which is introduced into river, lake or sea depends on its self-purification capacity and is a function of water quality demands in regard to the effluents. In many cities of the U.S.A., large quantities of primary effluents and digested sludge are released via pipes on the bottom of the sea at great distances from the shore into the open ocean without apparent harm. In near shore waters, especially bays, fjords, etc., sewages have to be treated so as to eliminate or reduce phosphates by electrolysis before introducing them into the sea; such treatment prevents unwelcome eutrophication. The degree and kind of pollution in the lower Elbe due to sewage from the city of Hamburg is discussed. The nutrient-rich Elbe water can be traced by $\mathrm{N}$ - and Pmeasurements all the way to the region near Helgoland and Amrumbank. In the Netherlands, construction of a pipeline for transporting wastes from a large strawboard and potato flour mill industry into the Eems estuary is being planned. The pollutional capacity of this industry has been estimated at approximately 12 million population equivalents during its autumn working season. Until 1975 six pipelines will transport sewages from the inner parts of the country to the shore. The influence of the Thames waters with their high $\mathrm{P}$ amounts on biological processes in English coastal waters is discussed. In view of recent developments and future plans, international agreements on marine pollution problems are urgently needed in order to prevent deleterious effects in coastal waters.
\end{abstract}

\section{EINLEITUNG}

Der Begriff „Marine Wasserverunreinigung“ ist vom Scientific Committee on Oceanic Research in Paris im Dezember 1966 als „Introduction by man of substances into the marine environment resulting in such deleterious effects as harm to living resources, hazard to human health, hindrance to marine activity including fishing and reduction of amenities" definiert worden.

${ }^{1}$ Meinem hochverehrten Lehrer, Herrn Prof. Dr. K. Günther, zum 60. Geburtstag gewidmet. 
"Marine Wasserverunreinigung" schließt alle durch den Menschen in das Meer eingeführten Stoffe ein, die eine schädigende Wirkung ausüben. Soweit es sich um industrielle Abfallstoffe handelt, triff diese Definition nahezu uneingeschränkt zu. Für häusliche Abwässer muß der Begriff jedoch etwas weiter gefaßt werden. Häusliches Abwasser ist charakterisiert durch einen hohen Gehalt an kohlenstoff-, stickstoff- und phosphorhaltigen Verbindungen und enthält Spurenelemente, Vitamine und Aminosäuren in relativ hohen Konzentrationen. Es ist durch eine „düngende“ Wirkung gekennzeichnet, die sich bei einer Einführung in das nährstoffarme Meer auf das stärkste in biologischer Weise bemerkbar macht. Die Auswirkungen auf die durch die lange Evolution eingeschaukelte Biozönose der See sind von höchst komplexer Natur und reichen von einer negativen bis zu einer eventuell sogar positiven Beeinflussung. Wasserverunreinigung durch häusliche Abwässer wirft das Problem der Eutrophierung auf, über deren Auswirkungen auf die Lebensgemeinschaft des Meeres, speziell in unserem Falle der Nordsee als eines flachen Randmeeres, bisher nur begrenzte Erfahrungen und Untersuchungen vorliegen.

Bei der Beurteilung der Auswirkungen häuslicher Abwässer auf die See befinden wir uns daher weitgehend auf Neuland. In vorliegender Arbeit soll versucht werden, einen Uberblick über die Herkunft und den Umfang der häuslichen Abwässer zu geben, die einmal direkt durch an der See gelegene Ortschaften und zum anderen indirekt durch die großen Flüsse dem Meer zugeführt werden. Ferner soll kurz über die Erfahrungen berichtet werden, die in den benachbarten, an der Nordsee gelegenen Ländern bei der Abwasserbeseitigung gemacht wurden, sowie über die Wege, die in Übersee, besonders in den USA und in Australien beschritten worden sind.

\section{ERGEBNISSE UND DISKUSSION}

Das Verfahren, Abwasser und Abwasserschlamm häuslicher Herkunft in größerem Umfang in das Meer oder in die Ästuare großer Flüsse einzuleiten, ist so alt wie die Geschichte der Abwasserbehandlung selbst. Vor über 100 Jahren wurde in London bereits damit begonnen, Abwasser in 4 großen Speicherbecken zu sammeln, um es nur bei Ebbe in die Themse abzuleiten. 1889 wurde dann eine Kläranlage gebaut, die eine primäre Abwasserbehandlung ermöglichte (FøYN 1965). Der in Absetzbecken zurückgehaltene Rohschlamm wurde mit 6 Schiffen auf See versenkt. Auch die Stadt Hamburg transportiert ihren ausgefaulten Klärschlamm mit Schiffen bis in die See. In New York setzen ebenfalls 5 der 7 neuen Kläranlagen Schlammtransportschiffe von $2000 \mathrm{~m}^{3}$ Ladeinhalt ein, die Abwasserschlamm in $20 \mathrm{~km}$ Entfernung von der Kïste ins Meer versenken (MüLLER 1953).

Fast ebenso alt wie die Bestrebungen, das Meer und die großen Ästuare für die Abwasserbeseitigung auszunutzen, sind die Bemühungen, die Folgen derartiger Einleitungen zu erkennen und Richtlinien aufzustellen, um unerwünschten Schäden vorzubeugen. Schon 1915 stellte WII.HELMI an der damaligen Königlichen Landesanstalt für Wasserhygiene in Berlin-Dahlem 31 Leitsätze für die Einleitung von Abwässern in das Meer auf (WILHELMI 1915), die grundsätzlich auch heute noch Gültigkeit besitzen.

Bis jetzt aber beschränkt sich die Kontrolle und Uberwachung von Abwasserein- 
leitungen in das Meer in Deutschland auf beratende und gutachtliche Tätigkeit staatlicher Oberbehörden. Es bleibt zu hoffen, daß in absehbarer Zeit Richtlinien erarbeitet werden können, die eine Grundlage für eine gesetzliche Regelung schaffen und nach dem Muster der für das Süßwasser schon lange in Kraft befindlichen Vorschriften die küstennahen Gewässer vor Abwasserschäden sichert. Letzten Endes ist eine internationale Regelung nach dem Beispiel der Olkonvention anzustreben.

Als Quellen der Verschmutzung durch häusliche Abwässer kommen einmal die direkt am Meer gelegenen Ortschaften und Städte in Betracht, zum anderen aber auch die großen Flüsse, die in ihrem Wasser eine beträchtliche Schmutzfracht aus dem Inland in mehr oder weniger abgebautem Zustand mitführen. Besonders ist an Elbe, Weser, Rhein und Themse zu denken mit ihren am Unterlauf gelegenen Großstädten Hamburg, Bremen, Rotterdam und London, deren Abwässer sich letzten Endes in der Nordsee auswirken.

Die Abwässer einer Stadt setzen sich aus häuslichen und gewerblichen Abwässern zusammen. Der charakteristische Bestandteil häuslicher Abwässer ist ihr hoher Gehalt an organischen, abbaufähigen Substanzen. Städtische Abwässer enthalten nach IMHofF (1966) im Mittel $600 \mathrm{~g}$ Gesamtschwebestoffe $/ \mathrm{m}^{3}$ und $660 \mathrm{~g}$ gelöste Stoffe $/ \mathrm{m}^{3}$ sowie einen Gesamtsauerstoffbedarf nach 5 Tagen von $360 \mathrm{~g} \mathrm{O}_{2} / \mathrm{m}^{3}$. In einem $\mathrm{m}^{3}$ sind im allgemeinen $80 \mathrm{~g}$ Stickstoff, $20 \mathrm{~g}$ Phosphorsäure als $\mathrm{P}_{2} \mathrm{O}_{5}$ und $60 \mathrm{~g} \mathrm{Kali}$ als $\mathrm{K}_{2} \mathrm{O}$ enthalten. Pro Einwohner fallen etwa 100 bis 2501 Abwasser pro Tag als Durchschnittswert an.

Im allgemeinen gelangen diese Abwässer über eine Kanalisation in eine Abwasserbehandlungsanlage. Die Reinigungsverfahren werden in drei Stufen unterteilt, von denen die erste Reinigungsstufe die mechanische Klärung durch Absetzen der Sinkstoffe umfaßt. In der zweiten Reinigungsstufe erfolgt die biologische Oxydation organischen Materials und in der dritten Reinigungsstufe werden die Nährsalze entfernt. Den $A b$ schluß einer vollständigen Reinigung kann eine Sterilisierung des Abflusses durch Chlorung bilden.

Das Ausmaß der Reinigung, das für ein Abwasser gefordert werden muß, ehe es in einen Vorfluter eingeleitet werden kann, hängt von der Selbstreinigungskraft eben dieses Vorfluters weitgehend ab. Abgeschlossene Meeresbuchten mit geringem Wasseraustausch oder flache Randmeere erfordern eine viel vorsichtigere Behandlung von $\mathrm{Ab}$ wassereinleitungen als der offene Ozean mit großen 'Tiefen und kräftigen Strömungen.

Als Beispiel für eine empfindliche Meeresbucht sei der Oslo-Fjord erwähnt (FøYN 1959), dessen innerer Teil durch die Abwässer der Stadt Oslo stark belastet ist. Die Abwässer machen sich an der Oberfläche durch Hypertrophierung und in der Tiefe durch ein starkes Sauerstoffdefizit mit $\mathrm{H}_{2} \mathrm{~S}$-Bildung bemerkbar. Um diese Verhältnisse langsam zu sanieren, wurde 1958 eine Versuchskläranlage mit einer dritten Reinigungsstufe erbaut, die dem Abwasser auf elektrolytischem Wege 90 bis $95 \%$ der Phosphate entzieht, die sonst als Minimumfaktor stark eutrophierend wirken würden. Wenn die Anlage den Erwartungen für eine längere Zeitdauer entspricht, soll das gesamte Osloer Abwasser nach diesem Prinzip in dritter Stufe gereinigt werden.

Dem einen Extrem der Reinigung häuslicher Abwässer bis zur dritten Reinigungsstufe vor Einleitung in das Meer sei das andere Extrem gegenübergestellt: die Einleitung überwiegend nur primär gereinigter Abwässer. Als Beispiel sei die Stadt Los 
Angeles herangezogen. Die Kläranlage der Stadt ist für einen Trockenwetterzufluß von $1600000 \mathrm{~m}^{3}$ Abwasser pro Tag ausgelegt. Ausgehend von ökonomischen Gesichtspunkten kam man hier zu dem Schluß, die Abwässer im wesentlichen nur primär gereinigt mit einer am Meeresboden verlegten Leitung soweit erforderlich vor der Küste direkt ins Meer zu pumpen (Abb. 1) (THeroux 1957). Berechnungen und großangelegte Versuche ergaben, daß die Leitung für das Abwasser eine Länge von $8 \mathrm{~km}$ haben muß. Sie mündet in $64 \mathrm{~m}$ Tiefe mit einem $y$-förmigen Verteiler. Die Ableitung des ausgefaulten Schlammes erfolgt über eine $11 \mathrm{~km}$ lange Leitung in $91 \mathrm{~m}$ Tiefe am Rande einer in

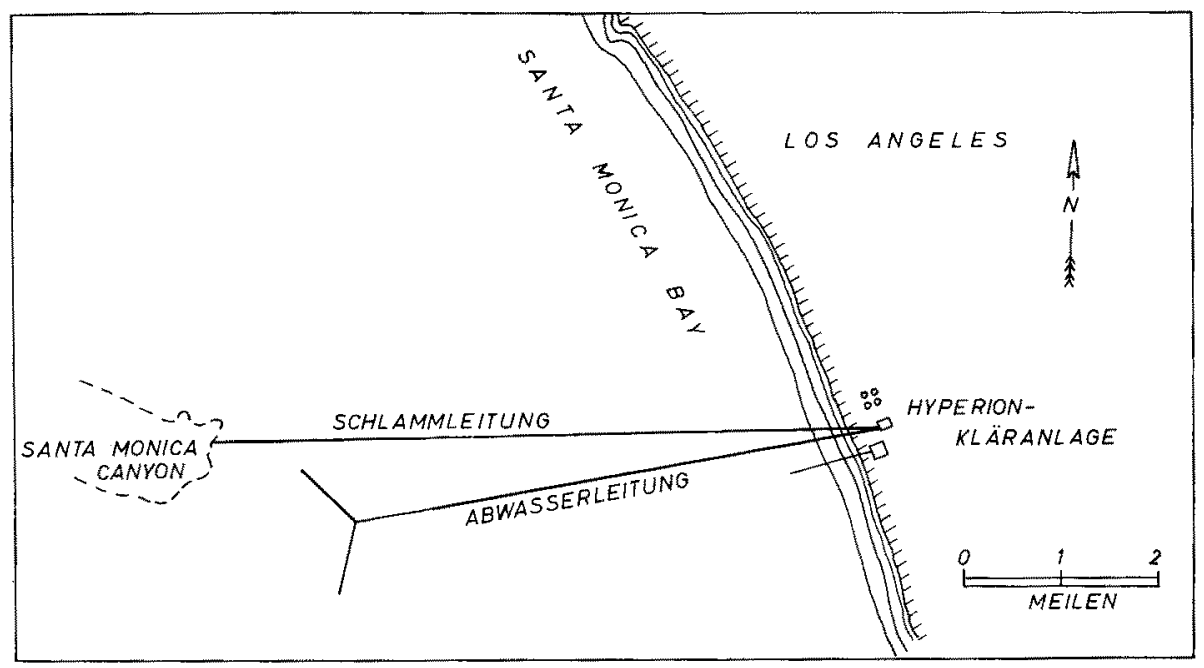

Abb. 1: Kläranlage bei Los Angeles. (Verändert nach THEroux 1957)

größere Tiefen abfallenden Rinne. Täglich werden 56 Tonnen Stickstoff als N, 26 Tonnen Phosphor als $\mathrm{PO}_{4}, 25$ Tonnen Silizium als $\mathrm{SiO}_{3}$ und $3000 \mathrm{~g}$ Vitamin $\mathrm{B}_{12}$ eingeleitet. Die Anlage ist seit 9 Jahren in Betrieb, ohne daß größere ökologische Veränderungen des Meeres nachgewiesen werden konnten. Die bakteriologischen Verhältnisse in der Bucht von St. Monica haben sich seit 1943 stark verbessert. (Hume \& Garber 1966, Koebig \& Koebig 1957-1962).

Weitere Beispiele bieten die Stadt San Diego (N.H. Brooks 1959), Orange County und Carlsbad in Californien, die in gleicher Weise primär gereinigte Abwässer über Rohrleitungen in die See einleiten (TrBby et al. 1964, Pomeroy 1959).

Die amerikanische Auffassung über eine Abwasserbeseitigung in See sei mit den Worten von Brooks zusammengefaßt: "A well-placed outfall with an effective diffuser may be much less costly than providing more thorough sewage treatment on land."

Ganz im Gegensatz zur amerikanischen Auffassung steht die Entwicklung der Abwasserbehandlung in Australien (MứLler 1959). Hier ist man bemüht, die Abwässer vor Einleitung in das Meer möglichst weitgehend zu reinigen. Adelaide behandelt seine Abwässer biologisch nach dem Belebungsverfahren, chlort sie zusätzlich noch während der langen Badesaison von Oktober bis April und leitet sie dann durch eine $300 \mathrm{~m}$ 
lange Rohrleitung vor der Küste in die See. In Perth ist ebenfalls eine Belebungsanlage für die biologische Reinigung im Bau.

Bei den amerikanischen und auch bei den australischen Beispielen dient der offene Ozean als Vorfluter für die Abwassereinleitungen. Er fällt relativ schnell vor der Küste in größere Tiefen $a b$ und bietet die Gewähr hinreichender Verdünnung.

Wenden wir uns nun wieder der Nordsee $z u$, so haben wir es mit einem flachen Randmeer zu tun, das durch mehrere große Flüsse kräftig eutrophiert wird. Die Ge-zeitenbewegung und starke Windturbulenz lassen eine feste Schichtung von Wasserkörpern im allgemeinen nicht $\mathrm{zu}$.

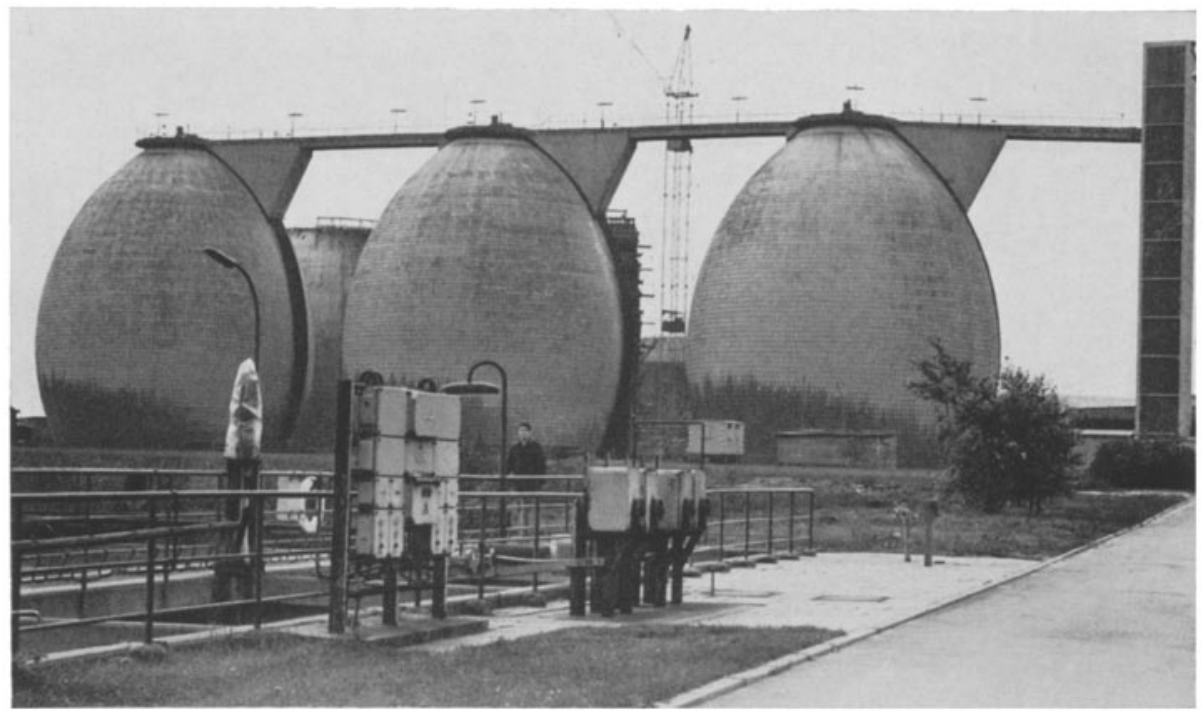

Abb. 2: Faultürme des Klärwerkes Köhlbrandhött in Hamburg. (Foto: Bildarchiy der Biologischen Anstalt Helgoland)

Als charakteristisches Beispiel einer Quelle der Verunreinigung der Nordsee sollen die Abwasserverhältnisse der Stadt Hamburg betrachtet werden. Hamburg hatte im Jahr 1966 einen Gesamtabwasseranfall von 200 Millionen $\mathrm{m}^{3}$, von denen 136,8 Millionen $\mathrm{m}^{3}$ teilbiologisch und 26,2 Millionen $\mathrm{m}^{3}$ vollbiologisch geklärt worden sind; 37,0 Millionen $\mathrm{m}^{3}$ Abwasser sind unbehandelt in den Vorfluter Elbe abgeleitet worden. Das Hauptklärwerk Köhlbrandhöft mußte einen Trockenwetterzufluß von $380000 \mathrm{~m}^{3} /$ Tag und einen Regenwetterzufluß von $450000 \mathrm{~m}^{3} /$ Tag bewältigen. Da die Anlage aber nur für einen Trockenwetterzufluß von $210000 \mathrm{~m}^{3} / \mathrm{Tag}$ gebaut wurde, blieb die Reinigung hinter der ursprünglich geplanten Leistung von 75 bis $80 \% \mathrm{BSB}_{5}$-Abbau zurück $\left(\mathrm{BSB}_{5}=\right.$ biochemischer Sauerstoffbedarf in 5 Tagen). Das Wasser, das im Einlauf mit

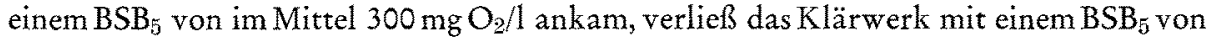
ca. $190 \mathrm{mg} \mathrm{O} / 1$, überschritt sogar häufig den Wert von $200 \mathrm{mg} \mathrm{O} / 1$. Um diese Verhältnisse zu verbessern, ist ein Ausbau des Klärwerkes Köhlbrandhö̈t auf $500000 \mathrm{~m}^{3} \mathrm{Ab}$ wasser/Tag geplant. Der Auslauf des Klärwerkes mündet am Köhlbrand in die Elbe. 
Das neue Klärwerk "Stellinger Moor" verarbeitet ca. $30000 \mathrm{~m}^{3} \mathrm{Abwasser/Tag}$ in vollbiologischem Verfahren. Im gesamten Süderelbegebiet einschließlich des Hafens werden aber noch alle Abwässer ungeklärt in die Süderelbe eingeleitet.

In der Schlammbehandlung des Klärwerkes Köhlbrandhöft, die nach dem Faulprinzip durchgeführt wird, werden die Faultürme (Abb. 2) mit 800 bis $1200 \mathrm{~m}^{3} / \mathrm{Tag}$ Frischschlamm beschickt. Der ausgefaulte Schlamm wird auf etwa $8 \%$ Feststoffgehalt eingedickt (Abb. 3) und auf ein Schiff verladen. Im Mittel fielen im Mai 1967 etwa 76 Tonnen Schlamm/Tag an, von denen 33 Tonnen auf mineralische und 43 Tonnen auf organische Bestandteile entfielen. Das Schiff übernimmt zusätzlich noch den aus-

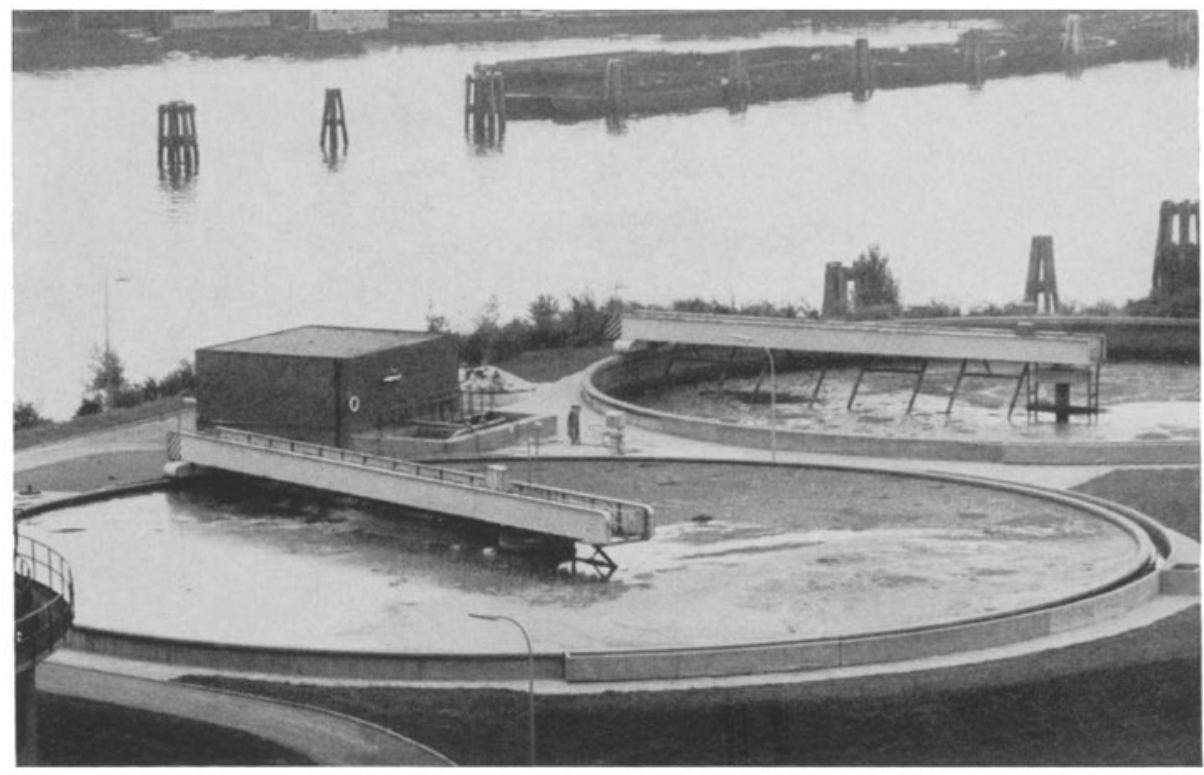

Abb. 3: Die Faulschlammeindicker des Klärwerkes Köhlbrandhöft in Hamburg. (Foto: Bildarchiv der Biologischen Anstalt Helgoland)

gefaulten Klärschlamm der Stadt Elmshorn, der in Mengen von $1000 \mathrm{~m}^{3} /$ Monat anfällt. Es befördert den Schlamm in das Gebiet von Feuerschiff Elbe I. Von der Elbmündung an muß ein Schlepper vorgespannt werden.

Der Schlamm sollte bei Feuerschiff Elbe I ursprünglich mit Hilfe eines Rohres in 6 bis $7 \mathrm{~m}$ Tiefe eingeleitet werden, zur Zeit wird er jedoch oberflächlich bei langsamer Vorausfahrt abgelassen, so daß eine breite, scharf konturierte schwarze Abwasserfahne im Meer entsteht. Die Schlammwolke scheint sich über eine längere Zeit relativ konstant zu erhalten. Anläßlich einer Besichtigungsfahrt im Juni 1967 war an der Einleitungsstelle nach einer Stunde immer noch deutlich ein $1,5 \mathrm{~km}$ langer und etwa $50 \mathrm{~m}$ breiter schwärzlicher Streifen zu erkennen.

Im folgenden soll nochmals die Elbe bei Hamburg betrachtet werden. Welches Schicksal erleiden die Abwässer Hamburgs auf ihrem Weg zur See und in welchem Zustand kommen sie letzten Endes in der Nordsee an? Hierzu gibt eine graphische Dar- 
stellung der „Behörde für Wirtschaft und Verkehr, Strom und Hafenbau“, (Hamburg 1964) einigen Aufschluß (Abb. 4). Die Abbildung zeigt einen Längsschnitt der Elbe von Schmilka an der tschechischen Grenze bis Cuxhaven für den August 1959. Im oberen Teil der Abbildung ist der Sauerstoffgehalt in $\%$ des Sättigungswertes aufgetragen. Auffallend ist die starke Sauerstoffzehrung von Meißen bis Magdeburg-'Tangermünde, die bis auf $25 \%$ Sättigung heruntergeht. Von der Zonengrenze bis Hamburg erholt sich die Elbe wieder bis zu einer Sauerstoffsättigung von $80 \%$, um im Hamburger Stadtgebiet erneut bis auf $25 \%$ abzusinken. Die Selbstreinigungskraft der Elbe ist

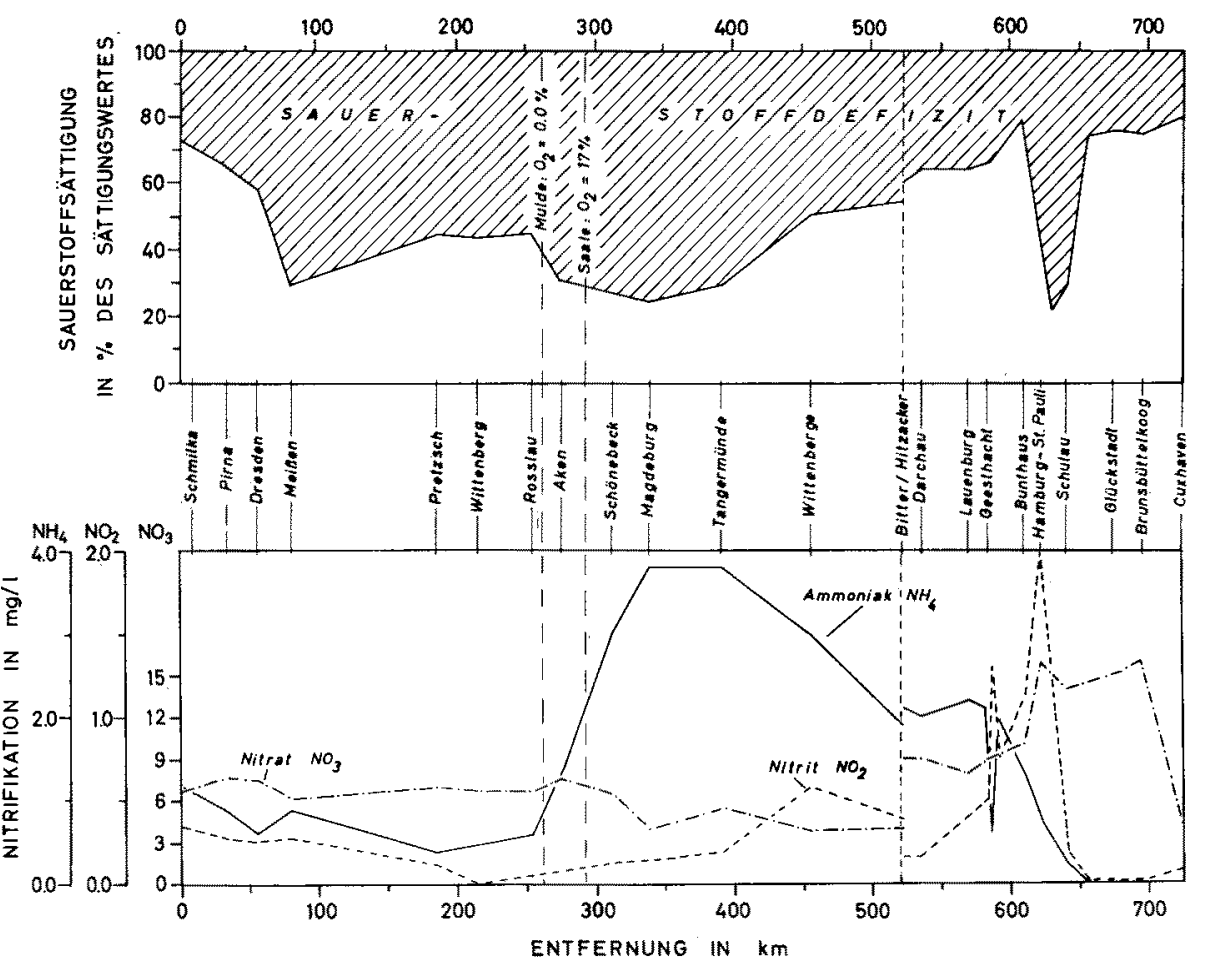

Abb. 4: Längsschnitt der Elbe von der tschechischen Grenze bis zur Mündung. (Verändert nach Behörde für Wirtschaft und Verkehr, Strom und Hafenbau, Hamburg 1964)

jedoch so groß, daß sie sich bis Stadersand wieder auf Werte von $75 \%$ Sättigung anreichert. Bei Cuxhaven sind $80 \%$ Sättigung erreicht. Die hier dargestellten Verhältnisse im Hamburger Stadtgebiet stellen einen extrem ungünstigen Zustand dar. Langjährige Untersuchungen der Elbe bei Teufelsbrück zeigen, daß es nur im Sommer oder bei anhaltender Eisbedeckung im Winter zu einer Unterschreitung einer 50\% Sauerstoffsättigung im Hamburger Stadtgebiet kommt. Anaerobe Zustände werden (nach Mitteilung des Hygienischen Institutes Hamburg) im freien Wasser praktisch nie erreicht.

Im unteren Teil der Abbildung 6 sind die Stickstoffkomponenten des Elbewassers getrennt nach $\mathrm{NH}_{4}{ }^{+}-$, $\mathrm{NO}_{2}{ }^{\prime}-$ und $\mathrm{NO}_{3}{ }^{\prime}$-Stickstoff aufgetragen. Speziell über die Elbe 
liegen hier weitere ausführliche Arbeiten von Kühl \& MANN (1958), UlKEN (1963), Lucht (1964) und RhenNHeImer (1965) vor, auf deren Ergebnisse hier Bezug genommen wird. Städtisches Rohabwasser enthält Stickstof teils in organisch gebundener Form, teils in Form von Ammoniumionen. Durch konventionelle biologische Klärverfahren oder durch die Selbstreinigungsprozesse im Fluß wird $\mathrm{NH}_{4}+$ in aerobem Milieu über $\mathrm{NO}_{2}{ }^{\prime}$ zu $\mathrm{NO}_{3}{ }^{\prime}$ bakteriell oxydiert. Im anaeroben Milieu können gegenläufige bakterielle Prozesse ablaufen, die vorhandenes $\mathrm{NO}_{3}{ }^{\prime}$ bis zum freien Stickstoff oder $\mathrm{NH}_{4}{ }^{+}$reduzieren können. Bestimmung der Stickstoffkomponenten geben uns daher ein gutes Bild von dem Stand der Auf- und Abbauvorgänge innerhalb des Stickstoffkreislaufes eines Gewässers.

In der Elbe betrug der $\mathrm{NH}_{4}^{+}$-Gehalt bei Eintritt in die Bundesrepublik im Jahresmittel $19604,3 \mathrm{mg} / \mathrm{l}$. In der Schleuse bei Geesthacht fanden lebhafte Stickstoffumsetzungen und Denitrifikationsvorgänge statt, wie sich aus dem unruhigen Kurvenverlauf (Abb. 4) erkennen läßt. In Hamburg kommt dann ein kräftiger Stoß stickstoffhaltiger Abwässer in die Elbe, die sich infolge der warmen Witterung im August 1959 sofort in lebhafter Nitrifikation bemerkbar machten. Dicht unterhalb Hamburgs lag ein hoher Nitritgipfel von ca. $2 \mathrm{mg} \mathrm{NO} / 1$, der von einem Gipfel der Nitrat-Werte mit ca. 15 mg $\mathrm{NO}_{3}^{\prime} / 1$ abgelöst wurde. Der Ammoniumgehalt fiel infolgedessen stark ab.

Die Selbstreinigung der Elbe ist nach etwa $30 \mathrm{~km}$ Fließstrecke unterhalb Hamburgs bei Schulau - Stadersand im großen und ganzen abgeschlossen. Durch die rechtselbischen Nebenflüsse Pinnau, Krückau und Stör, die zu den am höchsten belasteten Flüssen der Bundesrepublik gehören, kommt noch einmal eine starke Verschmutzung in die Elbe, die den $\mathrm{O}_{2}$-Gehalt des Hauptstromes bis auf $45 \%$ Sättigung erneut herabdrücken kann (MANN 1958).

Mit Eintritt in die Brackwasserzone zeigt die Elbe jedoch ein typisch $\beta$-mesosaprobes Bild. Aufgrund der mineralisierten Abwässer Hamburgs und der Oberelbe hat sich im Raum von Blankenese-Glückstadt ein reiches Süßwasserplankton entwickelt.

Von Glückstadt an macht sich ein langsam wachsender Salzgehalt bemerkbar, der durch die Gezeitenbewegung bedingt wird. Bei einem Salzgehalt von 2 bis $8 \%$ nimmt die Trübung des Elbewassers plötzlich stark zu. Die Längenausdehnung dieser Trübungswolke beträgt etwa 25 bis $30 \mathrm{~km}$. Sie wird auch in anderen, der Gezeitenbewegung ausgesetzten Flüssen beobachtet und entsteht nach PostMA \& KALLE (1955) durch eine Art "Sinkstoff-Falle". Eine Ablagerung der Trübungsstoffe erfolgt am stärksten in ruhigen Seitenarmen, Kanälen, Häfen und höher gelegenen Watten, zum Beispiel bei Brunsbüttelkoog, wo der Schlickfall nach Lucht (1964) 70 bis $80 \mathrm{~cm}$ im Monat beträgt. Zugleich finden gewaltige biologische Veränderungen in der Zusammensetzung der Lebensgemeinschaften des Flusses statt. In der Zone der großen Salzgehaltsschwankung bricht die Süßwasserbiozönose zusammen. Es kommt zu Absterbeprozessen, die ein Minimum der Phytoplanktongesellschaft in Gebiet Otterndorf-Medem herbeifuihren. Erneute Sauerstoffschwankungen und eine Zunahme der $\mathrm{NH}_{4}{ }^{+}$- und $\mathrm{NO}_{2}{ }^{-}$ Werte sind die Folge (Abb. 5) (MANN 1958). In der Abbildung können wir einen deutlichen $\mathrm{NH}_{4}{ }^{+}$-Gipfel von $8 \mathrm{mg} / \mathrm{l}$ bei Altenbruch-Cuxhaven sowie einen $\mathrm{NO}_{2}{ }^{\prime}$-Gipfel von $0,5 \mathrm{mg} / 1$ bei Otterndorf erkennen. Bei Altenbruch-Cuxhaven ist entsprechend ein $\mathrm{O}_{2}$-Einbruch aufgetreten. Bei Cuxhaven ist vermutlich in diesem Zusammenhang ein zweites Trübungsmaximum abgezeichnet. 
Das von der See her mit dem Gezeitenstrom eindringende Seewasserplankton geht ebenfalls in der Zone der starken Salzgehaltsschwankungen zugrunde. Erst vor der Flußmündung, etwa bei Feuerschiff Elbe III, kommt es wieder - aufgrund erneuter Mineralisation - zu einem Salzwasser-Phytoplankton-Maximum (Bursche, KüHL \& MANN 1958), das sich weiter nach See zu verdünnt.

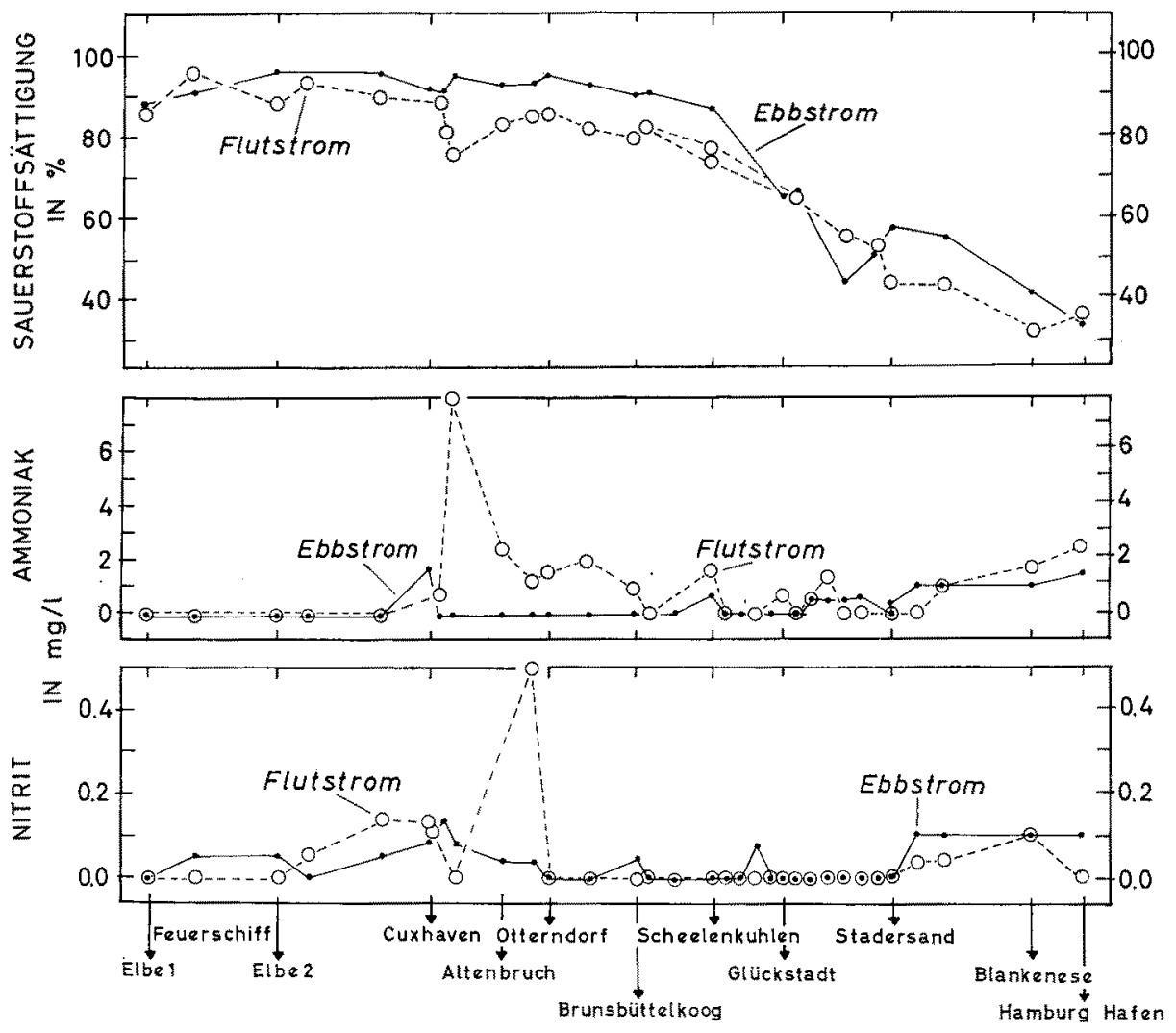

Abb. 5: Sauerstoffsättigung, Ammoniak- und Nitritgehalt der Elbe von Hamburg bis Feuerschiff Elbe I vom 16. bis 22. Oktober 1950. (Verändert nach ManN 1958)

Im Brackwasserbereich liegt also infolge der biochemischen und biologischen Umstellungen ein auch ohne anthropogene Einflüsse bereits belastetes System vor, wie CAsPERs (1967) ausführte. Der Fluß führt nicht nur Detritus und Abwasserreststoffe mit sich, sondern auch noch die gesamte abgestorbene Lebensgemeinschaft der Süßwasserzone und der von See her eindringenden Seewasserorganismen. Um die Flußmündung herum hat sich jedoch eine Lebensgemeinschaft angesiedelt, die auf die Verarbeitung dieses toten, absinkenden organischen Materials eingearbeitet ist. Weitere Untersuchungen werden zeigen, in welchem Maße dieser Lebensgemeinschaft noch eine zusätzliche Belastung mit aus dem Abwasser stammenden Reststoffen zugemutet werden kann. 
Die letzte große Verschmutzungsquelle im Elbeästuar stellt die Stadt Cuxhaven dar, die mit einem großen Anfall von Fischabwässern in ihrer Abwasserbehandlung noch nicht aus dem Planungstadium herausgekommen ist. Schätzungsweise werden ca. $20000 \mathrm{~m}^{3}$ Abwässer pro Tag ungereinigt an der Alten Liebe eingeleitet. Die Stelle markiert sich durch einen umfangreichen Möwenschwarm.

Folgt man dem weiteren Verlauf des Elbewassers in der Nordsee, so muß man sich darüber im klaren sein, daß der Abbau organischer Schmutzstoffe im Meer etwa um die Hälfte verlangsamt verläuft, während die Selbstreinigung von bakterieller Verschmutzung schneller vor sich geht als im Süßwasser. Im Meer sterben täglich $90 \%$ der vorhandenen Coli-Bakterien ab, im Süßwasser dagegen werden mehrere Tage benötigt (Mann 1958).

Der weitere Einfluß des Elbewassers auf die südliche Nordsee kann an Hand der Untersuchungen der Biologischen Anstalt Helgoland erläutert werden. Eine Schnittfahrt wird monatlich von Cuxhaven an seewärts bis Helgoland und darüber hinaus bis zum Feuerschiff P 8 durchgeführt und umfaßt 15 Stationen (Abb. 6). In den als Beispiel hier dargestellten Fahrtabschnitten vom 10. bis 11. Mai 1966 und 6. Januar 1967 kommt das Flußwasser bei Otterndorf-Cuxhaven mit Werten von $200 \gamma \mathrm{NH}_{4}+/, 54 \gamma$ $\mathrm{NO}_{2}{ }^{\prime} / 1,850 \gamma \mathrm{NO}_{3}{ }^{\prime} / 1$ und $100 \gamma \mathrm{PO}_{4} / 1$ an. Erst bei Feuerschiff Elbe I sind die Werte auf $100 \gamma \mathrm{NH}_{4}+/ 1,10 \gamma \mathrm{NO}_{2}^{\prime} / 1,300 \gamma \mathrm{NO}_{3}^{\prime} / 1$ und $25 \gamma \mathrm{PO}_{4}{ }^{\prime \prime \prime} / 1$ abgesunken und fallen nach See zu weiter $a b$. Auffällig ist, daß der Phosphatgehalt dicht hinter Cuxhaven bei Station 7 nochmals eine Zunahme auf $160 \gamma \mathrm{PO}_{4}{ }^{\prime \prime \prime} / 1$ zeigt. Diese Zunahme ist vermutlich auf Mineralisationsprozesse vor der Elbmündung zurückzuführen. Gleiches kann für die Nitrat- und Nitritgipfel bei Station 6 angenommen werden. Die vorliegende graphische Darstellung stellt jedoch nur die Ergebnisse weniger Fahrten dar. Weitere Auswertungen der über mehrere Jahre bereits durchgeführten Messungen werden zu einem späteren Zeitpunkt veröffentlicht werden.

Nach GillBRicht (1961) vermischt sich das Elbwasser nicht direkt mit dem Nordseewasser, sondern zunächst mit dem Wasser des Wattengebietes, das seinerseits ein Mischwasser aus reinem Nordseewasser und einem von der Elbe schon beeinflußten Küstenwasser darstellt. Ein guter Indikator ist auch die Keimzahl, mit der GunKeL (1963) Wasserinseln mit stark abweichendem Keimgehalt gelegentlich weit in See bis Helgoland beobachten konnte. Auch LüNEBurg (1963) konnte anhand der Phosphatverteilung den Einfluß der großen Ströme nachweisen. Küstenwasser mit Werten von $25 \gamma \mathrm{PO}_{4}^{\prime \prime \prime} / 1$ bei Feuerschiff Elbe I und Leuchtturm Roter Sand nehmen nach der offenen See zu bis auf Werte von $10 \gamma \mathrm{PO}_{4}{ }^{\prime \prime \prime} / 1$ bei Helgoland und Feuerschiff P 12 ab. Entsprechende Beobachtungen liegen von KREY (1956) für das Seegebiet vor der schleswigholsteinischen Westküste vor. Anhand des anorganischen Phosphors kann der Elbeeinfluß fingerförmig in Richtung Amrumbank verfolgt werden (Abb. 7).

Über die Hydrochemie der Weser geben uns die Untersuchungen von KüHL \& Mann (1958) und Bursche, Kühl \& Mann (1959) gute Aufschlïsse. Stärkere Abwasserbelastung des Unterlaufes ist nur im Raum Bremerhaven-Nordenham-Brake

Abb. 6: Verteilung von Salzgehalt, Temperatur, Ammonium-, Nitrit- und Nitratstickstoff und Phosphatgehalt auf einem Schnitt von Feuerschiff P 8 uber Helgoland bis Cuxhaven-Otterndorf vom 10. und 11. Mai 1966 und 6. Januar 1967 
Häusliche Abwässer und die Verunreinigung der Nordsee
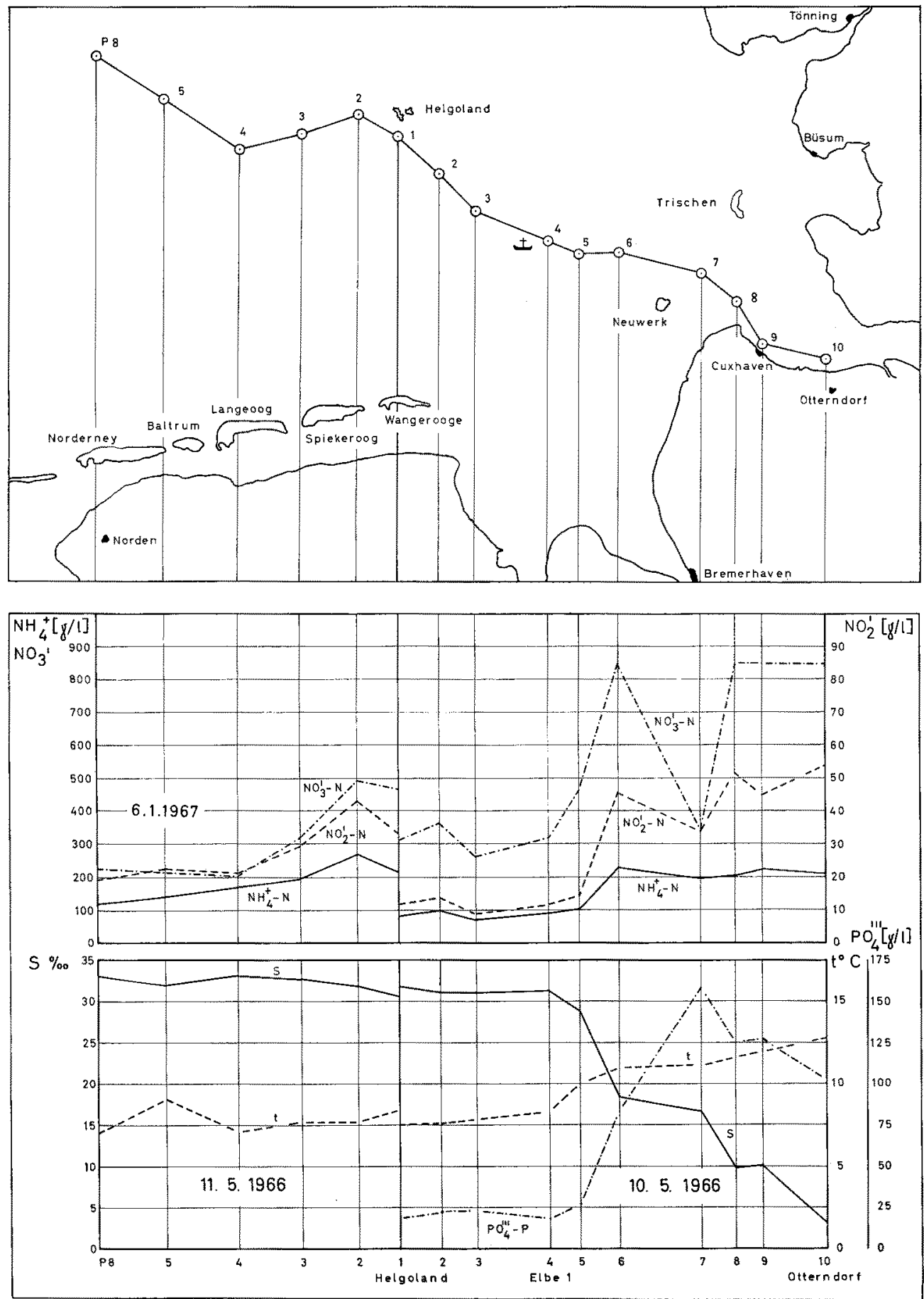
nachzuweisen, wo die Sauerstoffsättigung im Sommer bis auf ca. $55 \%$ zurückgehen kann. Beim Weserfeuerschiff steigt sie wieder auf $100 \%$ und mehr an. Das Sauerstoffminimum entspricht einem Trübungsmaximum. Die Längenausdehnung der Trïbungswolke beträgt rund $30 \mathrm{~km}$ und wird mit der Tidebewegung um 10 bis $15 \mathrm{~km}$ verschoben. Von den Stickstoffverbindungen tritt Ammonium in geringen Mengen von 1 bis $2 \mathrm{mg} / \mathrm{l}$ in der Unterweserstrecke auf und ist in der Gegend unterhalb Nordenhams nicht mehr nachzuweisen. Nitrit steigt nicht über $0,2 \mathrm{mg} /$, während die Nitratwerte mit 20 bis $25 \mathrm{mg} / \mathrm{l}$ relativ hoch liegen; nach See zu nehmen sie jedoch schnell $\mathrm{ab}$ und erreichen bei Weser-Fenerschiff nur noch 2 bis $3 \mathrm{mg} / \mathrm{l}$.

Über die Ems liegr eine ausführliche Arbeit von KüHL \& ManN (1955) vor, derzufolge keine wesentliche Abwasserbelastung im Unterlauf zu erkennen ist. Die Sauerstoffsättigung beträgt bei Leerort $80 \%$ und steigt emsabwärts bis auf $100 \%$ und mehr an. Der Ammoniumgehalt überschreitet nicht 1 bis $2 \mathrm{mg} / \mathrm{l}$ und auch die Nitratwerte liegen im Süßwasserbereich nur bei 6 bis $7 \mathrm{mg} / \mathrm{l}$, nach See zu abfallend. Eine Trübungszone ist im oberen Bereich der Brackwasserzone zwischen Leerort und Pogum ausgebildet.

Elbe-, Weser- und Emsmündung werden in einer kartenmäßigen Übersicht der Bundeswasserstraßen von 1961, herausgegeben von der Bundesanstalt für Gewässerkunde, als mäßig verschmutzt angegeben (HrRsCH 1961).

An größeren Städten liegen in diesem Gebiet Emden, das seine Abwässer teils grob-mechanisch, teils aber auch biologisch gereinigt einleitet, Norden mit biologischer Kläranlage und Wilhelmshaven mit überwiegend grob-mechanischer Klärung.

Von großer Bedeutung ist die Entwicklung der Abwasserverhältnisse in Holland. In der Provinz Groningen entsteht eine große Strohpappen- und Kartoffelmehl-Industrie, deren Abwässer über eine Entfernung von $32 \mathrm{~km}$ in das Emsästuar abgeleitet werden sollen (EGGINK 1966). Die Abwässer einer Kartoffelerntesaison werden etwa 12000000 Einwohnergleichwerten entsprechen. Die Abwässer sollen über 3 Pipelines mit einer täglichen Ladung von etwa 1,4 Millionen Einwohnergleichwerten in das Astuar gepumpt werden. In Zukunft wird mit einem weiteren Anwachsen dieser Industrie auf 13 Millionen Einwohnergleichwerte während einer Kampagne gerechnet. Zur Zeit wird bereits ein Teil dieses Abwassers über Kanäle in das Astuar befördert.

Im folgenden soll das Mïndungsgebiet von Rhein, Maas und Schelde betrachtet werden. Im Mittel führen alle drei Flüsse zusammen etwa $2400 \mathrm{~m}^{3}$ Wasser pro sec. (Kalle 1953). Der Rhein ist bekanntermaßen durch die ca. 40 Millionen Anwohner an seinen Ufern und durch die geballte Großindustrie schwer verschmutzt. Nach BoLoMEY (1959) ist an seiner Mündung am nördlichen Ufer eine Abwasserfahne ausgebildet. Die Sauerstoffsättigung liegt unterhalb $50 \%$. Bei Ebbe strömt das verschmutzte Flußwasser aus der Flußmündung hinaus in die See. Bei auflaufendem Wasser wird dieses Wasser in nördlicher Richtung an die Küste gedrückt und verschmutzt hier in bedenklicher Weise die großen Badestrände und Erholungsgebiete bis zum Seebad Scheveningen. Versuche mit Schwimmkörpern haben ergeben, daß selbst bei ablandigem Wind das Flußwasser für einige Stunden in jeder Tide an die Küste gedrückt wird. Erst bei Hochwasser staut das Seewasser dann den Rhein in die Flußmündung zurück, so daß wieder klares Salzwasser an der Küste entlangströmt.

Wie EgGINK im Frühjahr 1967 auf dem Kolloquium „Abwassereinflüsse in 


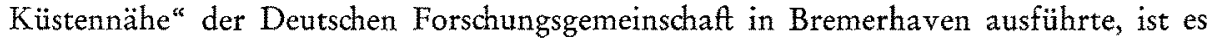
weiterhin geplant, auch für die Abwässer von Den Haag und den umliegenden Gemeinden mit zusammen etwa 1,5 Millionen Einwohnergleichwerten das Meer als Vor-

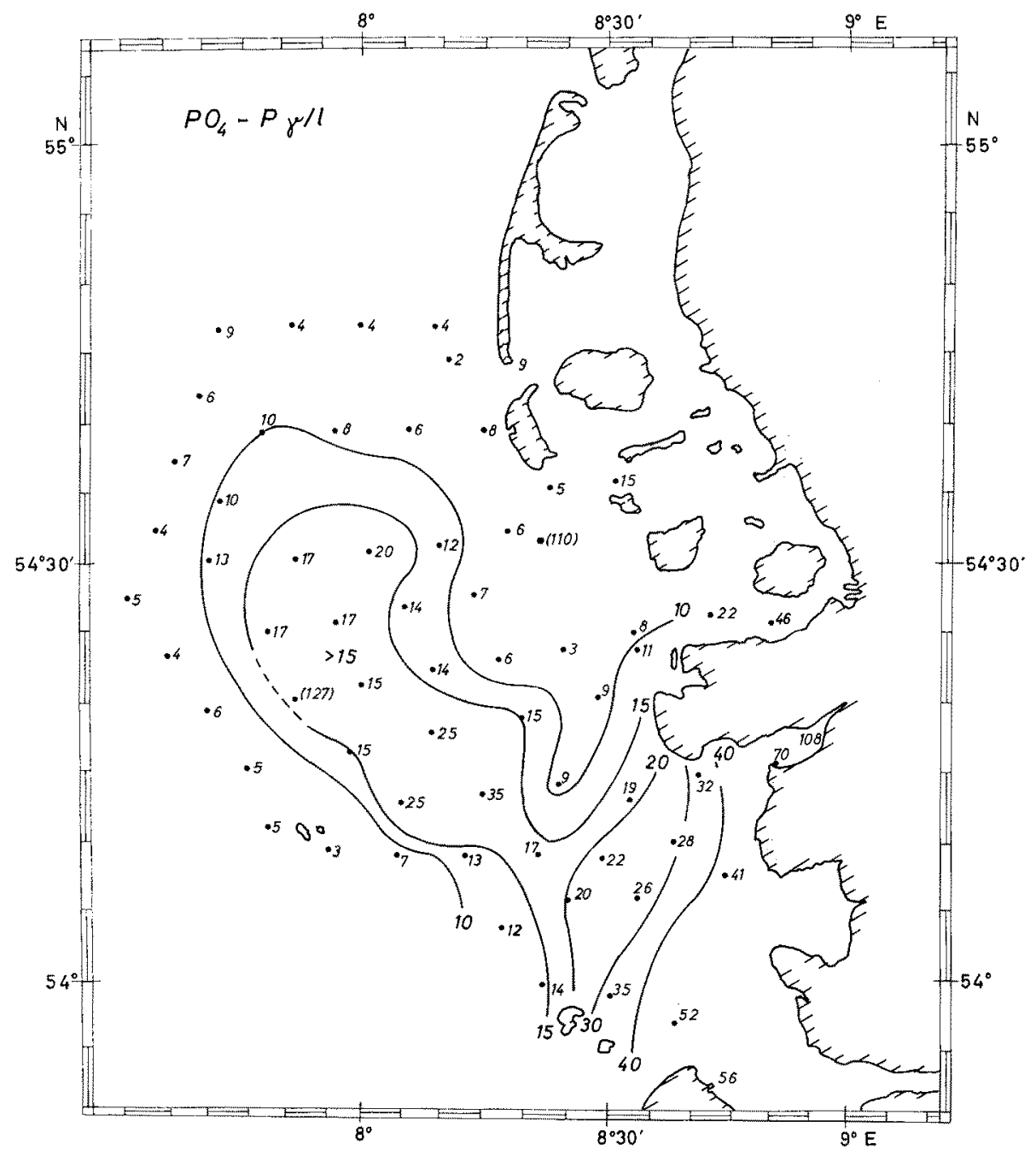

Abb. 7: Phosphatverteilung im Seegebiet vor der schleswig-holsteinischen Westküste im August 1954. (Verändert nach KREY 1956)

fluter auszunutzen. Ebenso soll ein Teil der Abwässer der Provinz Nord-Brabant mit Rohrleitungen in das Scheldeästuar abgeleitet werden. Ab 1975 sollen 6 Rohrleitungen an der ganzen Küste Hollands Abwässer aus dem Inland in das Meer abführen.

Wie KorRinga (1967) auf derselben Tagung darlegte, ist es erforderlich, vor allem den Küstenstreifen des Meeres vor Abfallstoffen zu schützen. In den flachen Wasser- 
körpern der holländischen Wattgebiete ist es stellenweise bereits zu starker Hypertrophierung durch Abwassereinführung gekommen, die zu Massenvermehrung einzelner Protozoenarten geführt haben. Eine giftige Wasserblüte wurde im Jahr 1961 durch den Dinoflagellaten Prorocentrum micans gebildet. Der Flagellat reicherte sich in den Muschelkulturen in hohem Maße an. Nach dem Genuß dieser Muscheln kam es bei verschiedenen Personen zu paralytischen Krankheitserscheinungen. Ahnliche Vergiftungen sind aus Portugal und Amerika berichtet worden.

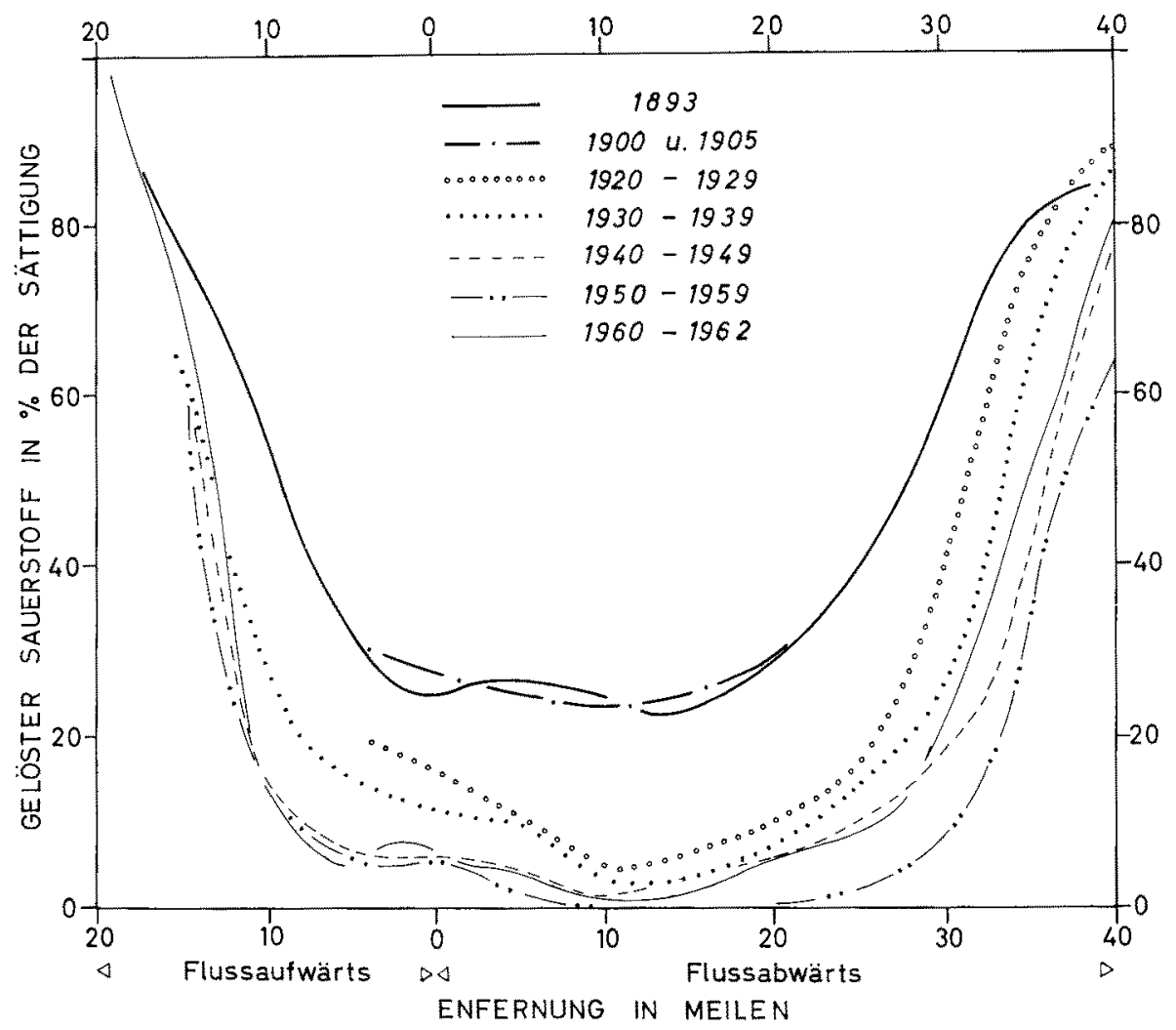

Abb. 8: Sauerstoffverteilung im Themseästuar während der Sommermonate von 1893 bis 1962. (Verändert nach Gameson, BarReTt \& Preddy 1964)

Bei der Untersuchung der Verschmutzung der Nordsee soll zum Abschluß die britische Insel betrachtet werden. Die Themse stellt durch die Abwässer der Stadt London das am stärksten belastete Astuar dar. Im Meßpunkt London Bridge wurde der Sauerstoffgehalt seit 1893 ständig verfolgt. Ausführliche Untersuchungsergebnisse liegen von Gameson, Barrett \& Preddy (1964), Gameson (1964) und Gameson \& Hart (1966) vor. Abbildung 8 zeigt die Verschlechterung der Sauerstoffverhältnisse in den letzten 60 Jahren. 20 Meilen oberhalb London Bridge kommt die Themse mit etwa $100 \%$ $\mathrm{O}_{2}$-Sättigung an. Die Abwässer Londons bewirken eine Zehrung, die bis zu völligem 
Verschwinden des Sauerstoffes führen kann. In den Jahren 1950 bis 1959 beispielsweise hatte die sauerstofffreie Zone in der Themse eine Längenausdehnung von ca. 10 Meilen. In der Zone wurde $\mathrm{H}_{2} \mathrm{~S}$ nachgewiesen. Der Ammoniumgehalt steigt im Sommer von 2 p.p.m. auf 8 p.p.m. an. Anfang des Jahres 1964 wurde die biologische Kläranlage der "Crossness Sewage Treatment Works" in Betrieb genommen. Man hofft damit, den Sauerstoff im Fluß ständig auf $5 \%$ Sättigung zu halten und damit jegliche $\mathrm{H}_{2} \mathrm{~S}$-Bildung im freien Wasser zu unterbinden. Als Erfolg ist zu verzeichnen, daß im Jahre 1965 von 2000 Proben nur noch 2 sauerstofffrei gefunden wurden. Letzten Endes wird die vollbiologische Reinigung der gesamten $A b$ wässer Londons angestrebt.

Im Mersey-Astuar zeigt sich ebenfalls ein starkes Sauerstoffdefizit bis zum vollständigen Sauerstoffschwund bei Widnes.

Die gleiche Entwicklung zeigt das Tees-Astuar. Es wird durch Industrieabwässer und Rohabwässer besonders im mittleren Teil belastet, so daß die Verteilung der Organismen der V-förmigen Kurve des Sauerstoffgehaltes entspricht (Department of Scientific and Industrial Research 1961).

Über die Auswirkungen des Themsewassers auf die südliche Nordsee liegt eine Arbeit von Kalle (1953) vor. Kalle macht es anhand einer Phosphorbilanz deutlich, wie stark sich die eutrophierende Wirkung bis in die See hinein bemerkbar macht. Bei einem Phosphorgehalt von $3 \mathrm{gP} / \mathrm{m}^{3}$ in der Themse ergibt sich bei einer mittleren Wasserführung von $67 \mathrm{~m}^{3} / \mathrm{sec}$ ein Eintrag von 17,3 Tonnen P/Tag. Dies führt zu einer P-Akkumulation in einem verhältnismäßig ruhigen Wasserkörper im Südosten Englands, im Stromschatten des Kanals, mit Werten von etwa $10 \gamma \mathrm{P} / 1$ als Gesamtphosphor. Hierdurch ergibt sich eine erhöhte Trophie. KaldE führt den Mehrertrag der Fischerei in den Hoofden im Vergleich zu den benachbarten Gebieten auf diese Nährstoffanreicherung zurück.

Anhand der letzten Betrachtung über die eutrophierende Wirkung der Themse sei nochmals auf die eingangs erwähnte Definition des Begriffes „Marine Wasserverunreinigung" hingewiesen. In bezug auf die häuslichen Abwasseranteile müssen die Auswirkungen sowohl negativ als auch vielleicht positiv bewertet werden. Noch ist zu wenig bekannt über die Zusammenhänge des Stoffwechselgeschehens, um generell jegliche Abwassereinleitung in See untersagen zu können. Eine Tatsache bleibt die wachsende Bevölkerung und Industrialisierung der Küstengebiete, deren Abwasserprobleme gelöst werden müssen. Wegweisend scheinen hier unsere holländischen Nachbarn vorzugehen, die bei ähnlich gelagerter Küstensituation uns in der Bewältigung des Programms „Meer als Vorfluter für Abwässer" um einige Jahre Praxis voraus sind.

\section{ZUSAMMENFASSUNG}

1. Häusliche Abwässer sind durch einen hohen Gehalt an C-, N- und P-haltigen Substanzen, Spurenelementen und wichtigen organischen Verbindungen, wie Vitaminen und Aminosäuren, gekennzeichnet. Im Stoffhaushalt der relativ nährstoffarmen See können sich diese Verbindungen in negativer wie auch in positiver Weise bemerkbar machen. 
2. Die Verschmutzungsquellen des Meeres durch häusliche Abwässer können ihrer Herkunft nach in 2 Gruppen unterteilt werden: (a) Abwassereinleitungen aus direkt am Meer gelegenen Ortschaften, (b) das Wasser großer Ströme, das Abwässer und Abwasserinhaltsstoffe in mehr oder weniger abgebautem Zustand aus dem Innern des Landes mit sich führt.

3. Der Grad der Reinigung, der an ein Abwasser gestellt werden muß, bevor es in einen Vorfluter eingeleitet werden kann, hängt von der Selbstreinigungskraft des Vorfluters ab und von den Ansprüchen, die an den Vorfluter gestellt werden müssen.

4. Am Beispiel amerikanischer Verhältnisse wird gezeigt, daß die Einleitung großer Mengen nur primär gereinigter Abwässer mit Hilfe von Rohrleitungen in größerem Abstand vor der Küste ein ökonomisches Verfahren der Abwasserbeseitigung darstellt, da der offene Ozean schnell in größere Tiefen abfällt und eine hinreichende Verdünnung der Abwässer gewährleistet ist.

5. Das Beispiel des Oslo-Fjordes zeigt, daß für abgeschlossene Meeresbuchten eine Reinigung der Abwässer bis zur dritten Stufe vor Einleitung in See erforderlich werden kann, um einer unerwünschten Eutrophierung der Küstenzone vorzubeugen.

6. Anhand der Abwasserverhälnisse der Stadt Hamburg wird die Belastung der Unterelbe diskutiert. Der Einfluß des nährstoffreichen Süßwassers kann an der Verteilung der $\mathrm{N}$ - und P-Komponenten bis über Helgoland und Amrumbank hinaus in See verfolgt werden.

7. Auf holländischer Seite wird der Bau einer Strohpappen- und Kartoffelmehlindustrie für 13 Millionen Einwohnergleichwerte pro Erntesaison geplant, deren $\mathrm{Ab}$ wässer in das Emsästuar eingeleitet werden sollen.

8. In Holland sollen bis 1975 sechs Rohrleitungen gelegt werden, die Abwässer aus dem Inneren des Landes direkt zur Küste befördern werden.

9. Der eutrophierende Einfluß der Themse auf die südliche Nordsee wird am Beispiel einer Phosphorbilanz nach einer Arbeit von KaLle (1953) dargestellt. Die Eutrophierung wirkt sich hier in einer Steigerung der Fischereierträge aus.

10. Angesichts der gegenwärtigen Situation und der für die unmittelbare Zukunft in Aussicht genommenen Maßnahmen ist eine gesetzliche, internationale Regelung über die Belastungsgrenzen von Meeres- und Küstengebieten durch häusliche $\mathrm{Ab}$ wässer dringend erforderlich, um diese Gebiete vor Schäden zu schützen.

\section{ZITIERTE LITERATUR}

Bolomey, J. G. W., 1959. Effect of the Rhine on Netherlands beaches. In: Proceedings of the 1st International Conference on waste disposal in the marine environment. Ed. by E. A. Pearson, Oxford, Pergamon Press, 164-174.

Brooks, N. H., 1959. Diffusion of sewage effluent in an ocean-current. In: Proceedings of the 1st International Conference on waste disposal in the marine environment. Ed. by E. A. Pearson, Oxford, Pergamon Press, 246-267.

Bursche, E. M., Kühl, H. \& ManN, H., 1958. Hydrochemie und Phytoplankton in der Unterelbe. Veröff. Inst. Meeresforsch. Bremerh. 5, 165-192.

- 1959. Beziehungen zwischen dem Chemismus und der Phytoplanktonentwicklung auf der unteren Weser. Int. Revue gesamt. Hydrobiol. 44, 277-298. 
CASPERs, H., 1967. Biologische Gesichtspunkte der marinen Abwasserbelastung. In: Bericht über das Colloquium Abwassereinflüsse in Küstennäbe der Deutschen Forschungsgemeinschaft in Bremerhaven vom 17.-18. März 1967, 12-15.

Croft, J. E., 1960. Pollution of coastal and esturial waters. J. Proc. Inst. Sew. Purif. Pt 4 $431-438$.

Department of Scientific and Industrial Research, 1961. Pollution of estuaries. Pt 1. Effects of pollution. (Notes on water pollution, No. 11.) J. Proc. Inst. Sew. Purif. Pt 3, 253-256.

EgGrNk, H. J., 1966. Predicted effects of future discharges of industrial wastes into the Eems estuary, In: (Proceedings of the) $3 \mathrm{rd}$ International Conference on water pollution research. Munich, 1966. Sect. 3. Paper No. 1, 1-20 (Preprint). [Ersch. bei: Oldenbourg, München.]

- 1967. Die Aufgabe des Reichsinstitutes für Abwasserreinigung in Holland; die Richtlinie betreffs Abflüsse in das nahe Meer. In: Bericht über das Colloquium Abwassereinfliisse in Küstennäbe der Deutschen Forschungsgemeinschaft in Bremerhaven v. 17.-18. März 1967, $71-73$

Foyn, 1959. Chemical and biological aspects of sewage disposal in inner Oslofjord. In: Proceedings of the 1st International Conference on waste disposal in the marine environment. Ed. by. E. A. Pearson. Oxford, Pergamon Press, 279-284.

- 1965. Disposal of waste in the marine environment and the pollution of the sea. Oceanogr. mar. Biol. Rev. 3, 95-114.

Gameson, A. L. H., 1964. Pollution of London's river. New Scient. 22, 295-298.

- Barretr, M. J. \& Preddy, W. S., 1964. Predicting the condition of a polluted estuary. In: Advances in water pollution research. Proceedings of the 2 nd International Conference in Tokyo, Aug. 1964. Vol. 3. Ed. by E. A. Pearson. Oxford, Pergamon Press, 167-177.

- \& HarT, I. C., 1966. A study of pollution in the Thames estuary. Chemy Ind. 44, $2117-2123$.

Gillbricht, M., 1961. Eine Methode zum direkten Nachweis von Ammoniak im Seewasser. Helgoländer zeiss. Meeresunters. 8, 58-67.

Gunkel, W., 1963. Daten zur Bakterienverteilung in der Nordsee. Veröff. Inst. Meeresforsch. Bremerh. (Sonderbd) 1, 80-89.

HrRsch, A., 1961. Die Verschmutzung der Bundeswasserstraßen (ohne Seewasserstraßen) eine kartenmäßige Übersicht ïber den gegenwärtigen Zustand und die hauptsächlichen unmittelbaren Abwassereinleiter. Dt. gewëasserk. Mitt. 5, 126-127.

Hume, N. B. \& Garber, W. F., 1966. Marine disposal of digested screened wastewater solids. In: (Proceedings of the) 3rd Conference on water pollution research, Munich 1966. Sect. 3. Paper No 12 (Preprint). [Ersch, bei: Oldenbourg, München.]

IMHoff, K., 1966. Taschenbuch der Stadtentwässerung. 21. Aufl. R. Oldenbourg, München, 368 pp.

Kalle, K., 1953. Der Einfluß des englischen Küstenwassers auf den Chemismus der Wasserkörper in der südlichen Nordsee. Ber. dt. wiss. Kommn Meeresforsch. 13, 130-135.

Koebig \& Koebig, Inc. Engineering-Architecture, 1957-1962. Sewage treatment and disposal for City of Los Angeles, $90 \mathrm{pp}$.

Korringa, P., 1967. Vortrag auf dem Colloquium Abroassereinflüsse in Küstennühe der Deutschen Forschungsgemeinschaft in Bremerhaven y. 17.-18. März 1967. Anhang zum Bericht.

KREY, J., 1956. Die Trophie küstennaher Meeresgebiete. Kieler Meeresforsch. 12, 46-64.

KüHL, H. \& ManN, H., 1955. Über die Hydrochemie der unteren Ems. Veröff. Inst. Meeresforsch. Bremerb. 3, 126-158.

- 1958a. Beiträge zur Hydrachemie der unteren Weser. Veröff. Inst. Meeresforsch. Bremerb. 5, 34-62.

- 1958b. Das Verhalten anorganischer Stickstoffverbindungen im Mündungsgebiet eines Flusses. Arch. FischWiss, 9, 9-16.

Luchr, F., 1964. Hydrographie des Elbe-Ästuars, hydrographische und hydrochemische Verhältnisse im Mündungsbereich der Elbe mit Einschluß des angrenzenden Oberlaufes. Arch. Hydrobiol. (Suppl. Bd) 29 (2), 1-96. 
LüNEBURG, H., 1963. Wassermischvorgänge vor der Weser- und Elbmündung. Veröff. Inst. Meeresforsch. Bremerh. 8, 111-141.

ManN, H., 1958. Abwasserprobleme in Tideflüssen. Ber. abwassertech. Verein, H. 9, 1-12.

MÜLler, W., 1953. Die Einleitung von Abwässern ins Meer. Gesundheitsingenieur 74, 286-288.

- 1959. Anforderungen an die Reinigung der Abwässer in Australien. Gas- $u$. WassFach 100 (48), Ausg. Wasser-Abwasser 635, 1247-1249.

Pomeroy, R., 1959. The empirical approach for determining the required length of an ocean outfall. In: Proceedings of the 1st International Conference on waste disposal in the marine environment Ed. by E. A. Pearson. Oxford, Pergamon Press, 268-278.

Postma, H. \& Kalle, K., 1955. Die Entstehung von Trübungszonen im Unterlauf der Flüsse, speziell im Hinblick auf die Verhältnisse in der Unterelbe. Dt. bydrogr. Z. 8, 137-144.

Rheinhermer, G., 1965. Mikrobiologische Untersuchungen in der Elbe zwischen Schnakenburg und Cuxhaven. Arch. Hydrobiol. (Suppl. Bd) 29 (2), 181-251.

Theroux, R. J., 1957. Enlarged sewage disposal facilities for Los Angeles. Sewage ind. Wastes 29, 124-133.

Tibby, R. B., Foxworthy, J. E., OGURI, M. \& FAy, R. C., 1965. The diffusion of wastes in open coastal waters and their effects on primary biological productivity. In: Pollutions marines par les microorganismes et les produits petroliers. Symposium de Monaco, Avril 1964. Ed. par la Commission Internationale pour l'Exploration Scientifique de la Mer Méditerranée, Monaco. Sécrétariat Général de la Commission, Paris, 95-113.

UlKeN, A., 1963. Die Herkunft des Nitrits in der Elbe. Arch. Hydrobiol. 59, 486-501.

Verschmutzung der Elbe und ihrer Nebenflüsse sowie die Bemühungen, sie rein zu halten. Uber die, 1964. Behörde für Wirtschaft und Verkehr, Strom und Hafenbau, Hamburg. (Mitteilung des Senats an die Bürgerschaft 6/65 Anl.)

Wilhelmi, J., 1915. Leitsätze für die Einleitung von Abwässern in das Meer. Wass. Abraass. 9 (15), 513-517.

\section{Diskussion im Anschluß an den Vortrag KAYSER}

MANN: Bei der Darstellung hygrographischer Untersuchungen und der Aufstellung von Abwasserlastplänen geht man oftmals nur von den Durchschnittswerten aus. Für die Gewinnung eines Überblicks über die vorliegenden Verhältnisse in einem Gewässer ist das sicher richtig und notwendig. Als Biologen müssen wir aber fordern, daß wir auch die Extremwerte heranziehen. Sie können das Vorkommen oder Fehlen von Tierarten bestimmen. Bei zusätzlichen Belastungen durch Abwässer soll man deshalb nicht von den Durchschnittswerten bei der Begutachtung ausgehen, sondern stets auch die möglichen Extremwerte heranziehen.

KAYSER: Ich stimme Hern Professor MANN voll zu und habe deshalb in meinem Vortrag am Beispiel der Elbe auch auf die Extremwerte des trockenen Sommers 1959 für die Darstellung der Belastung im Hamburger Raum zurückgegriffen.

FørN: Dr. KaYser hat über die Verhältnisse im Oslofjord gesprochen und nach den Resultaten der elektrolytischen Reinigungsmethode gefragt. $\mathrm{Da}$ ich für diese Methode verantwortlich bin, mödhte ich sagen, daß wir bis jetzt die Behörden in Oslo noch nicht überzeugen konnten, daß eine Reinigung notwendig ist. Wir haben aber Geld für eine wissenschaftliche Untersuchung bekommen, die wir nun abgeschlossen haben, und deren Resultate wir in diesem Symposion bekanntgeben wollen.

Posrma: Die natürliche Eutrophierung des Wattenmeeres, verursacht durch die Wirkung des Gebiets als "Trübungsfalle" oder Falle für organische Stoffe, kann man auf 50 bis 100 Tonnen P-Äquivalente pro Tag schätzen. Sie ist von derselben Größenordnung wie die Frachtabfuhr der Elbe, Weser und Ems (etwa 100 Tonnen P pro Tag). Deshalb ist es lokal of schwierig, $z$ wischen "natürlicher" und Abwassereutrophierung zu unterscheiden.

GERLACH: Wirkt sich die Eutrophierung der Elbmündung durch häusliche Abwässer positiv auf den Fischereiertrag aus? 
KAyser: Als direkte Folge der Eutrophierung durch häusliche Abwässer ist zunächst an eine Steigerung der Primärproduktion in der Elbmündung und in den davor gelegenen Seegebieten zu denken. Erst indirekt können dann auch die weiteren Glieder der Nahrungskette profitieren, als deren Endglied die Fische anzusehen sind. Da bekanntermaßen aber nur ein geringer Prozentsatz an Energie innerhalb der Nahrungskette weitergereicht wird, bleibt die Frage offen, ob die Menge der häuslichen Abwässer, die durch die Elbe zugeführt werden, überhaupt $\mathrm{zu}$ einer Steigerung des Fischereiertrags ausreichen.

CASpers: Die Verklappung der Hamburger Klärschlamm-Abfälle geschieht in einem Gebiet der Außenelbe, das durch natürliche Sedimentationsprozesse Schlickhalden enthält. Das hier vorhandene Benthos hat durch die Zuführung anthropogenen Schlammes keine wesentliche Beeinträchtigung erfahren. Ein gewisser zusätzlicher Düngungsfaktor scheint sich sogar positiv auszuwirken. Bei der Einbringung von häuslichem Abwasser in das Meer muß die Korrelation zu der natürlichen Sedimentation von Detritus berücksichtigt werden. 